\title{
TCM-Grid: Weaving a Medical Grid for Traditional Chinese Medicine
}

\author{
Huajun Chen, Zhaohui Wu, Chang Huang, and Jiefeng Xu \\ Grid Computing Lab,College of Computer Science, Zhejiang University,P.R.China, \\ \{huajunsir,wzh, changhuang, xujf\}@zju.edu.cn \\ http://grid.zju.edu.cn
}

\begin{abstract}
We present a TCM-Grid for Traditional Chinese Medicine (TCM). The purpose of the TCM-Grid is to aid the development of distributed systems that help health professionals, researchers, enterprizes and personal users to retrieve, integrate and share TCM information and knowledge from geographically decentralized TCM database resources and knowledge base resources in China. Our approach involves developing a Database Grid for discovering and accessing TCM database resources coordinately and a Knowledge Base Grid supporting TCM knowledge sharing globally. With our application experience, we argue that nowadays' Grid architecture is not enough: we need Database Grid to support finely granular data sharing and integration, and we also need a Knowledge Base Grid to support knowledge-intensive task. We also recommend a Grid Ontology effort to enable Grid intelligence.
\end{abstract}

\section{Introduction}

Traditional Chinese Medicine (TCM), with a history that spans thousands of years, has provided us a wide variety of resources for biomedical and health science. These resources include TCM literatures, medicinal materials, Chinese herbs, TCM compounding rule, Chinese medical formula and so on, both ancient and present. In China, there have been thousands of TCM hospitals, enterprizes and research institutes who have developed various TCM information products to serve people's health care and research demands such as TCM self-health consultation, TCM literature analysis and retrieval, new medicine design,etc. For example,by cooperating with China Academy of Traditional Chinese Medicine, we have set up Traditional Chinese Medical Database System, a series of databases of TCM [10] .

In both e-business and e-science for TCM, we have an urgent need to integrate TCM systems or services across distributed, heterogeneous, dynamic "virtual organizations" formed from the disparate TCM resources within a single organization (hospitals, enterprizes and research institutes) and/or from external resource sharing and TCM information resource provider relationships. Fortunately, we have found that the OGSA (Open Grid Service Architecture) [1] has promised to addresses above challenges. Therefore, with the cooperation with China Academy of Traditional Chinese Medicine, we began to develop 
a TCM-Grid to achieve our goals. However, with our first inspection and efforts, we have found that OGSA is not enough to satisfy our real needs and expectations.

At first, OGSA dose not provide proper way of database resource integration, registration and discovery. Second, medical informatics is a knowledge-intensive domain, we have requirements of represent TCM information at a knowledge level and construct high-level expert system such as TCM new medicine design expert system and TCM tutor expert system,etc. That urged us to provide basic infrastructure supporting TCM knowledge base creation, maintaining, utilizing and knowledge sharing across China. At last, sometimes we want autonomic discovery, binding and then integration of resources, in other word, we need some intelligence to help us discover and locate proper resources (both database resources and knowledge base resources) in such a information overwhelming environment,but OGSA care nothing on that at all(dynamic dose not mean intelligence).

Therefore, we introduce an architecture in term of Database Grid to support smaller granularity data sharing (database resource sharing). And with our research on semantic web [11] area, we have also present an architecture in term of Knowledge Base Grid to support knowledge sharing in semantic web. We also argue that to bring the Grid into its full potential, we should develop Grid Ontology corresponding to the Web Ontology effort in Semantic Web communities to address the complexity, dynamic, diversity and heterogeneity of a Global Grid and support autonomic grid service discovering,locating and executing .

\section{The Backbone of TCM-Grid: DB-Grid and KB-Grid}

\subsection{Database Grid}

Comparing to the DataGrid [2] with the aim of the sharing of huge amounts of distributed data files that we call as coarsely granular data resources over the network infrastructure, the Database Grid (DB-Grid) initiative is to develop and test the technological infrastructure that will enable the sharing of databases that contain more finely granular data resources.

A large number of existing database are important information resources, which contain rich domain specific information. Take the TCM area as an example, through years of work on collection, translation and compilation of ancient books of TCM, hundreds of TCM databases have been formed which collects numerous clinic cases, medicine prescriptions, diseases records and other precious experience in the TCM field. These databases constitute a basic information platform that needs to be accessed in many TCM research activities. Database Grid should get each of these database resources accessible on the Grid. Furthermore, Database Grid should provide users with an efficient mechanism for coordinated use of these content-related databases, for example providing the integration access service by constructing a virtual database so that users can access distributed data sources with single access point. 
In a service-oriented view, we should respectively define some generic database services. These generic services define the functionalities that a qualified database resource must provide. In [3] we have proposed the following basic services:

- Database Grid Information Service: support the initial discovery and ongoing monitoring of the existence and characteristics of resources, services, computation and other entities

- Database statement service: support the typical operations on database contents, including retrieval, insertion, deletion, and modification of the data schema;

- Database Management Service : enables DBA to perform a remote control;

- Database Accounting Service : enables database users to be charged of resource usage;

- Database Directory : Database directory is an aggregate registry that collects, manages and indexes individual database information models;

- Virtual Database : A virtual database is a middleware that integrates a number of databases but does not actually store any data;

- Database Market : data market is an open place for databases to interchange database units.

\subsection{Knowledge Base Grid}

TCM and medical informatics is a knowledge-intensive domain. In a serviceoriented perspective, we want to build large-scale knowledge-based service such as self-health consultation services.Underlying those services are there a variety of TCM knowledge base resources such as TCM Ontology, TCM compounding rule bases, TCM therapeutic principle knowledge bases, etc., which maybe be maintained by different organizations and/or individuals all around China.

Nevertheless, how could we integrate so many decentralized knowledge base resources to support constructing intelligent services? We argue that OGSA is not enough here for its aim to integrate information systems but not knowledge-based systems. In the following, we extend this argument in two respects and then introduce the core components to define more precisely how our KB-Grid functions and how it could be implemented and applied.

\subsubsection{Standard Knowledge Representation for Web}

To overcome the heterogeneity of web,the first thing we need is a standard method for knowledge representation and information description in web. With this effort, we can enable the web to be an intelligent information space, which is machine-understandable. That's the aim of the W3C's activity on Semantic Web [5]. They have recognized the importance of the standardization of knowledge representation and recommended the RDF [5] as the basic model for web knowledge representation. Above the RDF, DAPPA has presented the DAML+OIL [12] for Web Ontology description. 
Based on above efforts, we have proposed a hybrid knowledge markup language (KML) as our standard method for TCM knowledge representation. KML consists of a D-Box (Description Logic Box) for TCM-Ontology definition, R-Box (rules box) for TCM compounding rule definition, and a C-Box (case knowledge box supporting case-based reasoning) for TCM clinical cases description. The KML schema draft has been available from our website (http://grid.zju.edu.cn).

\subsubsection{Standard Knowledge Protocols}

In an environment with intelligence enabled, that means there are great of knowledge services distributed on the Grid, those knowledge services must coordinate their activities with each other to further their own interests or satisfy group goals. The fundamental concerns lying here are distributed control and semantic interoperability, that means we need knowledge level protocols. The design of knowledge protocol is more difficult and complex than simple information protocols such as LDAP or SOAP. As all known, computing grid has chosen the LDAP protocol for their GRIP/GIIS [ [ ] , and maybe they will choose SOAP as the substitute. We argue that both LDAP and SOAP is not enough for knowledge level communication.

At first, at the knowledge level, the message format for communication should contain the knowledge interchanged. The knowledge interchange format is very different with the data model adopted in LDAP. It should take some form like the first order logic syntax at the semantic level. At second, the control process is more complex here for that we should model the BDI (Belief, Desire, Intention) of the intelligent services by epistemic logic. Those are topics of multi-agents system in distributed artificial intelligence. We have made some suggestion on designing the knowledge protocol for KB-Grid in [4]. Such knowledge protocols will play core role not only for knowledge system cooperation but also for autonomic application integration in large-scale environment.

\subsubsection{Core Components of KB-Grid}

A typical KB-Grid consist of the following core components:

Shared Ontologies: Ontology lay down the ground concepts and rules for their domain. One could view the ontology as some controlled vocabulary which could facilitate the semantic interoperability between heterogeneous knowledge base resources .[14]

Knowledge Server: Our Knowledge Server is the web container for knowledge which is represented by RDF/DAML+OIL/OWL/RuleML. It is the runtime environment for webKBs. The key characteristic of knowledge server is that it could process semantic query, then do some inference within its KBs and then return the answers in a semantic form.

KB-MDS: KB-MDS is provided for model the meta-information about the organization and discovery of the $\mathrm{KB}$ resources.It act as the meta-directory for webKBs 
Ontology Browser: that's the user interface for KB-Grid. It supports semantic browsing against the knowledge server. We have finished the prototype of a TCM-Ontology browser. It supports TCM-Ontology (represented by RDF) and semantic query against the TCM-Ontology server.

For more detail about KB-Grid, please refer 4].

\subsection{Grid Ontology}

The Web communities such as W3C has initialed a Web Ontology effort [13]. Ontologies figure prominently in the emerging Semantic Web as a way of representing the semantics of documents and enabling the semantics to be used by web applications and intelligent agents. Ontologies can prove very useful for a community as a way of structuring and defining the meaning of the metadata terms that are currently being collected and standardized.Ontologies are critical for applications that want to search across or merge information from diverse communities. Although XML DTDs and XML Schemas are sufficient for exchanging data between parties who have agreed to definitions beforehand, their lack of semantics prevent machines from reliably performing this task given new XML vocabularies. [7] tells us more about the importance and the usage of Ontology in Web.

We argue that OGSA will face the same problem when it scales up globally. We really have used an ontology for the Computing Grid. That ontology includes the concepts and vocabularies for computing grid domain, for example CPU, Memory, Storage device and their combination to construct high-level virtual machines. Yes, that is the MDS data model existing in the LDAP server. For Computing Grid, the data model for LDAP is enough, because the resource model of computing grid is simple. However, when we extend the Grid to global application or service integration, the data model of LDAP or SOAP will fail because the global web is really much more complex than we have imagined : so many heterogenous web resources (web databases, web pages, web services) cover almost all domain of human knowledge and distribute all around the world. To enable Grid intelligence, we need build Grid Ontology to enable autonomous service discovery and integration.

Generally, a domain of virtual community such as TCM will build their own shared Ontology. Then the organization and/or individual who want to join such community will obey that Ontology to build their information or knowledge systems. Section3 will introduce the TCM Ontology which we have built for TCM community.

\section{Implementation of TCM-Grid}

To verify the ideas presented above, this section will discuss some key issue with respect to the implementation of TCM-Grid. 


\subsection{TCM Ontology}

The Ontology we want to build include related terms/concepts, their definitions and/or meanings, their relationships with each other, and some basic rules of the domain. We use Protg 2000, which is developed by Medical Informatics of Stanford University, to build TCM ontology. Generally, Protg 2000 uses the Semantic Web language RDFS as basic data storage format, but it can be easily adapted and extended to the other Semantic Web languages such as DAML+OIL.

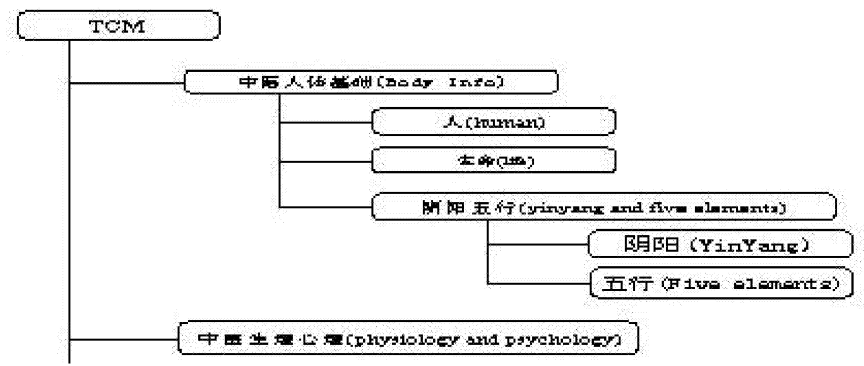

Fig. 1. The snapshot of TCM Ontology

Fig. 1 depicts the snapshot of TCM ontology class hierarchy. It shows that TCM is an abstract base class in TCM Ontology, and there are about 13 basic slots (attributes) of it. Then there exist about 20 subclasses like Human Info, psychology and physiology, therapeutics, and Chinese Medical Formula in TCM Ontology. TCM ontology has contained much more special concepts and classes such as yin-yang symptom complex, five-elements, body point, Chinese Medical Formula, Meridians, etc. Now we have finished building the whole class definition of TCM Ontology and edited about 100,000 records of TCM Ontology instances.

UMLS (Unified Medical Language System) is a web-accessible medical thesaurus developed by NLM (National Library of Medicine) of America as a basic knowledge source in medicine. It has 135 kinds of semantic classes and about 53 semantic relationships defined. Because TCM is one of the members of world medicine, we adopt this formal and regular definition in medicine as the basis of TCM ontology design.

\subsection{TCM-Grid Infrastructure}

Just as the figure2 has illustrated, we divide the TCM-Grid infrastructure into three levels. The backbone consists of a variety of data sources and knowledge sources. Above those sources, we could construct high-level services including some high-level database service and knowledge-based services. Above those services, we could form the virtual hospitals, virtual enterprizes and virtual research institutes. 


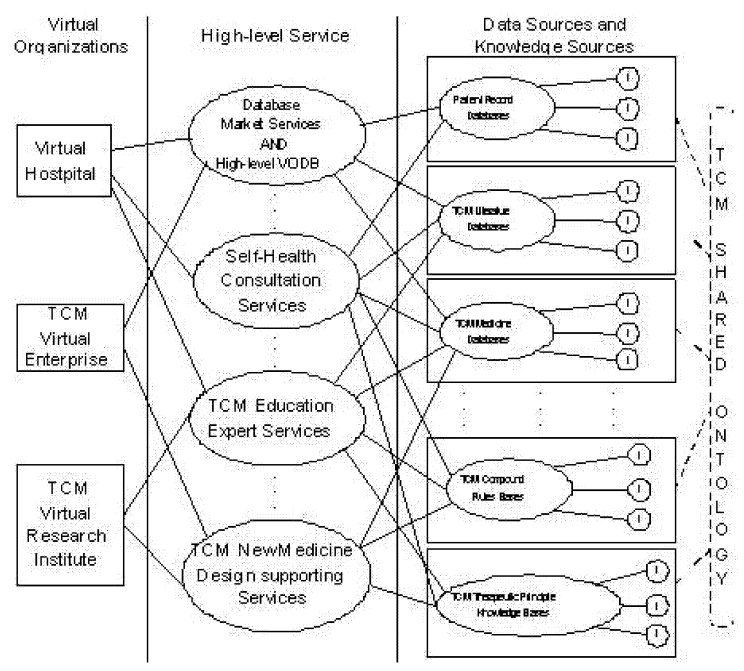

Fig. 2. TCM-Grid Infrastructure

Database Grid is used to manage database resources in a TCM virtual oraginzation. In this case, there are a number of basic databases, including $\mathrm{Pa}$ tient Record Databases, TCM Medicine Databases, TCM OTC Databases, TCM Literature Databases, Traditional Chinese Drug Database, Traditional Tibetan Drug Database and so on. Those databases serve as public information sources, which are geographically distributed and owned by different institutions and/or individuals. For example, the patient record databases maybe consist of hundreds of databases belonging to different hospitals distributed all around China, and we could call such a database group a sub-db-grid of TCM-Grid. Above that, a virtual database is constructed to integrate those basic TCM databases and provide federated access service. One can access one another's local data in two modes. One way is through the database access service, which deals with remote requests and return records. The other is downloading the application data units from a database market and loading them into its own database for local access. We have finished the design of the database resource model, and are developing a component which could run as daemon at the database client, when some modifications (adding a table for example) have happened in the member database, it will update the information about himself in the central database directory server.We are also developing the virtual database component which act as a uniform DB-Grid query interface. User sends their query to the virtual database service, and the virtual database service will dispatch the query to proper member databases by the meta-information stored by the central directory server.

KB-Grid is used to facilitate knowledge sharing among TCM VOs. In this case, there are also a number of knowledge bases including TCM symptom KBs, TCM Therapeutic Principle KBs, TCM medical formulas, TCM Com- 
pound Rule Bases, TCM Therapeutic Case Bases and so on. The TCM Ontology is also a kind of knowledge source, and it includes the TCM metathesaurus and TCM specialist lexicon. The TCM metathesaurus contains information about TCM concepts and terms from many controlled vocabularies and classifications used in patient records, administrative health data, bibliographic and full-text databases and TCM expert systems. The TCM specialist lexicon provides the lexical information needed for the specialist Natural Language Processing. We have finished the building of TCM ontology (about 10M). That ontology has contained a hierarchical TCM terminology represented by RDF/RDFS. Almost all the synonyms in TCM have been also included. We are developing a TCM ontology browser that has two main functionalities: the first one is the graphical display and semantic browsing of the ontology; the second is that it acts as the ontology-base query answer user interface.

Relying on these basic data and knowledge sources, we could construct high-level service. Moreover, above the high-level services, we could form virtual organizations.

\subsection{A Working Scenario: TCM New Medicine Design}

To make our discussion more clearly, we give a simple working scenario for TCMGrid.

Relying on the basic data and knowledge sources, a clinical development research for a new medicine is carried out, which can be deployed into the following phrases: TCM compound rule analysis, new medicine and medical formula design, preclinical testing, clinical testing and new medicine production. The above process maybe involves a research institute, a few hospitals for clinical test and some enterprizes to produce and sell the new medicine. At first, the research institute initiates a new medicine design process. With the assistance of the new medicine design supporting service, they make analysis of the TCM compound rule base and TCM therapeutic principle knowledge base, and design a new TCM medical formula. Some hospitals are involved in the preclinical test and clinical test phrase. With their clinical decision supporting service, they make the clinical test and record the results. When the new medicine is appropriate, some TCM enterprise will be involved and put the new medicine into production.

We have seen a working scenario which have a urgent need for dynamic integration of geographically distributed database resources, information system and knowledge-based system across the Internet environment.

\section{Related Work}

For database resources sharing across Grid, DAI-WG (Database Access and Integration Working Group) in GGF [8] is currently identifying consistent and effective ways of making existing, autonomously managed databases available within a Grid setting. Most requirements identified by them are applied to our proposal. Their approach to database access and integration is constructing OGSA 
compatible service specification. However, we think protocol is a fundamental issue in defining any networked computing model no matter what form of implementation is finally taken. So our efforts will focus on the formal representation of the related protocols.

For the large-scale web-based knowledge-base system , The Knowledge Grid effort in Chinese Academy of Sciences [9] has also proposed a worldwide resource (knowledge, information, and services) sharing and management platform. However, we argue that their platform has no awareness of developing knowledge protocols and high-level knowledge services. Another work worthy to mention is the DARPA's DAML-S 12] effort which supplies Web service providers with a core set of markup language constructs for describing the properties and capabilities of their Web services in unambiguous, computer-intepretable form. DAML-S markup of Web services will facilitate the automation of Web service tasks including automated Web service discovery, execution, interoperation, composition and execution.

\section{Conclusion and Future Work}

This paper describes our experience with building a medical grid for Traditional Chinese Medicine. Our ultimate goal is to aid the development of distributed systems that help health professionals, researchers, enterprizes and personal users to retrieve, integrate and share TCM information and knowledge from geographically decentralized TCM database resources and knowledge base resource all around China.

Based on our experience, we have found that nowadays' Grid architecture such as OGSA does not satisfy our real need and expectations. With our effort on Database Grid and Knowledge Base Grid, three respects have enhanced the OGSA. First, the DB-Grid enhances OGSA by supporting the database resources sharing by its database services and protocols, because most of the e-businesses involve the database. Second, KB-Grid enhances OGSA by knowledge sharing and knowledge-based services . Third, Grid Ontology enhances OGSA for its usage as the central control vocabulary which will enables the automatic Grid service dicovering, locating and executing.

In the future, we plan to finish the design and implementation of core database services and protocols and then deploy those components to the Traditional Chinese Medical Database System 10. In the knowledge-based system aspect, we plan to design and implement a knowledge server as the run time environment for RDF-based webKBs, and high-level knowledge query language will be designed to support ontology query . All the above efforts will be integrated into the TCM-Grid effort.

Acknowledgement. We gratefully acknowledge helpful discussion with other members in the Grid Computing Lab of Zhejiang University. This work is supported in part by the Grid-Based TCM Dynamic Information Resource Management and Knowledge Service subprogram of the Foundational Technology and Research Program, China Department of Science and Technology, and the China 
863 Research Program on Intelligent Workflow Technologies supporting Creditable E-Commerce under Contract 2001AA414320, and the China 863 Research Program on Core Workflow Technologies supporting Components-library-based Coordinated Software Development under Contract 2001AA113142.

\section{References}

1. The Physiology of the Grid: An Open Grid Services Architecture for Distributed Systems Integration. I. Foster, C. Kesselman, J. Nick, S. Tuecke, Open Grid Service Infrastructure WG, Global Grid Forum, June 22, 2002.

2. The Data Grid: Towards an Architecture for the Distributed Management and Analysis of Large Scientific Datasets. A. Chervenak, I. Foster, C. Kesselman, C. Salisbury, S. Tuecke. Journal of Network and Computer Applications, 23:187-200, 2001.

3. Zhaohui Wu, Changhuang, Guozhou Zhen, Database Grid: An Internet Oriented Database Resource Management Architecture, in Proceedings of GCC 2002, the International Workshop on Grid and Cooperative Computing of Chinese Academic of Science..

4. Zhaohui Wu, Huajun Chen, Jiefeng Xu, The Anatomy of Knowledge Base Grid, in Proceedings of GCC 2002, the International Workshop on Grid and Cooperative Computing of Chinese Academic of Science.

5. W3C Semantic Web Activity:http://www.w3.org/2001/sw/Activity [6]

6. Karl Czajkowski, Steven Fitzgerald, Ian Foster, Carl Kesselman : Grid Information Services for Distributed Resource Sharing: Proc. 10th IEEE International Symposium on High-Performance Distributed Computing (HPDC-10),IEEE Press,2001;

7. Deborah L. McGuinness. "Ontologies Come of Age". In Dieter Fensel, J im Hendler, Henry Lieberman, and Wolfgang Wahlster, editors. Spinning the Semantic Web: Bringing the World Wide Web to Its Full Potential. MIT Press, 2002.

8. Database Access and Integration Services WG of GGF, website, http://www.ggf.org/6_DATA/dais.htm;

9. H.Zhuge, A Knowledge Grid Model and Platform for Global Knowledge Sharing, Expert Systems with Applications, vol.22, no.4, 2002, pp.313-320, (Elsevier Science), 2002.

10. Traditional Chinese Medical Database System ,website, http://www.cintcm.com/e_cintcm/index.htm;

11. Tim Berners-Lee, James Hendler, Ora Lassila, the Semantic Web, Scientific American, May 2001

12. The DARPA Agent Markup Language Program: http://www.daml.org/

13. W3C Web-Ontology Activity: http://www.w3c.org/2001/sw/WebOnt/

14. Stanford Knowledge Interchange Format: http://www-ksl.stanford.edu/knowledge-sharing/kif/ 